\title{
The Relation between Diabetes Type 1 and 2 Type and Microalbuminuria in Omdurman Teaching Hospital and Altyser Health Center by Using Urine Strip for Microalbuminuria in Sudanese Patients
}

\author{
Nazik Ibrahim Abdel Rahman \\ Faculty of Medicine, Alzaiem Alazhari University, Sudan
}

Copyright $\bigcirc 2018$ by authors, all rights reserved. Authors agree that this article remains permanently open access under the terms of the Creative Commons Attribution License 4.0 International License

\begin{abstract}
The present work is Prospective cross sectional study. The main objective is to determine the presence of microalbuminuria in the patients urine in relation to the duration and the age and gender by using urine strip for microalbuminuria. The complications of diabetes mellitus especially end stage renal failure is a major health problem, This study is about the screening diabetic patients for microalbuminuria by simple urine strip for microalbuminuria. During period from Jan. - Aug. 2008, 100 diabetic patients were randomly selected from diabetic clinics of Omdurman Teaching Hospital and Altyser Health Center by using simple direct standardized questionnaire for 100 diabetic patients type I \& II. And the urine samples were examined for microalbuminuria Results: out of 100 diabetic patients, $66 \%$ were females and $34 \%$ were males, type I constitutes about $5 \%$ and type II about 95\%, the ages range from (9-90) years. Microalbuminuria detected in about $41 \%$ of the patients and $59 \%$ had negative results, most of the patients with positive results were in age group $(45-54)$. The clinical practice in diabetic clinics in Sudan is not following the international guidelines regarding the screening of diabetic nephropathy, so a significant number of the diabetic patients with diabetic nephropathy are missed.
\end{abstract}

Keywords Diabetes, Mellitus, Microalbuminuria

\section{Introduction}

Diabetes is worldwide in distribution and the incidence of both types $1 \& 2$ diabetes is raising, it was estimated that in the year 2000, 150 million people worldwide had diabetes, and this is expected to double by $2010^{1}$. This global pandemic principally involves type 2 and is associated with several contributory factors including increasing long life, obesity, unsatisfactory diet, sedentary lifestyle and increased urbanization. The prevalence of both types of diabetes varies considerably around the world and is related to differences in genetic and environmental factors.

(Type 1 diabetes) or (type 2 diabetes) due to relative deficiency of insulin results in decreased anabolic and increased catabolic effects. In Both type 1 and type 2 diabetes, the action of insulin are also impaired by insensitivity of target tissues, while this is a fundamental defect in type 2 diabetes ,hyperglycemia can also reduce insulin secretion by the effect of glucose toxicity on beta cell function leading to many complications, Hypoglycemia, Diabetic Retinopathy, Diabetic Nephropathy and Diabetic Neuropathy.

Proteinuria \& Microalbuminuria ${ }^{2}$ : Proteinuria is an abnormally high amount of protein in the urine. Proteins in the blood, like albumin and immunoglobulin, help coagulation (clotting), balance bodily fluids, and fight infection. The kidneys remove wastes from protein-rich blood through millions of tiny filtering screens called glomeruli. Most proteins are too large to pass through the glomeruli into the urine. The glomeruli are negatively charged, so they repel the negatively charged proteins. Thus, a size and charge barrier keeps protein molecules from entering the urine. Age and weight gain also increase the risk.

Signs and Symptoms: Foamy urine and swelling (edema) are two signs of Proteinuria that become more evident as the disease progresses. Excess protein can cause the urine to foam in water. This occurs because protein changes the surface tension between urine and water. Edema usually only occurs in nephritic range Proteinuria.

Albumin is particularly useful in absorbing bodily fluid into the blood. Because the albumin molecule is relatively 
small, it is often among the first proteins to enter the urine after glomeruli are damaged. Therefore, even minor kidney dysfunction is detectable with proper diagnosis of microalbuminuria. Reduced albumin level in the blood causes fluid retention and swelling that is first noticeable in the hands, lower legs, and feet. In more serious cases, the abdomen and face may swell.

Orthostatic Proteinuria is a disorder seen occasionally in children and young adults who leak significant amounts of protein when they are upright (orthostatic). Presumably, standing increases the pressure on the glomeruli and causes more protein to enter the urine, while lying down relieves pressure and causes less protein leakage. This is a benign disorder that most young people outgrow.

Diagnosis of Microalbuminuria ${ }^{3}$ Proteinuria and Microalbuminuria are diagnosed by measuring the amount of protein excreted in the urine in a 24-hour period. Collecting urine for 24 hours can be difficult, so the physician often uses a spot urine test in which one sample of urine is analyzed. Healthy kidneys continually remove creatinine from the blood to maintain a stasis between blood levels and urine levels. The ratio of protein to creatinine in the urine can provide the physician with a good idea of how much protein is being excreted over 24 hours.

Albumin can be measured in the urine at relatively low amounts, which allows the physician to diagnose patients with early glomerular damage. In people without diabetes, more than $1000 \mathrm{mg}$ of protein in the urine in a 24-hour collection may warrant blood analysis and, possibly, a kidney biopsy.

Treatment; It is necessary to treat the underlying condition that is causing Proteinuria. For example, when diabetes or hypertension is present, treatment involves controlling blood pressure with the use of an angiotensin-converting enzyme (ACE) inhibitor, like captopril. ACE inhibitors prevent the conversion of angiotensin I to angiotensin II, a substance that normally causes the veins to constrict. The result is a reduction in blood pressure.

The goal is to reduce blood pressure below 130 systolic and 80 diastolic. After BUN, creatinine, and potassium are monitored for 7 to 10 days, ACE inhibitors are gradually increased to avoid side effects. The degree of Proteinuria is checked periodically and typically improves with every dose.

Microalbuminuria Urine Test: A micro albumin test checks urine for the presence of albumin. Albumin is normally found in the blood and filtered by the kidneys. When the kidneys are working properly, albumin is not present in the urine. When the kidneys are damaged, small amounts of albumin leak into the urine. This condition is called microalbuminuria.

Microalbuminuria is most often caused by kidney damage from diabetes. However, many other conditions can lead to kidney damage, such as high blood pressure, heart failure, cirrhosis, or systemic lupus erythematous (SLE). If early kidney damage is not treated, larger amounts of albumin and protein may leak into the urine. This condition is called macroalbuminuria or Proteinuria. When the kidneys spill protein, it can mean serious kidney damage is present. This can lead to chronic kidney disease. A micro albumin urine test can be done on a sample of urine collected randomly (usually after the first time you urinate in the morning), a sample collected over a 24-hour period, or a sample collected over a specific period of time, such as 4 hours or overnight.

Nephropathy in diabetes: Diabetes has become the most common single cause of end-stage renal disease (ESRD) in the U.S. and Europe; this is due to the facts that

1) Diabetes, particularly type 2 , is increasing in prevalence;

2) Diabetes patients now live longer;

3) Patients with diabetic ESRD are now being accepted for treatment in ESRD programs .

Types: The following five types of Proteinuria are distinguished by milligrams $(\mathrm{mg})$ of protein measured during a 24-hour urine collection:
1. Microalbuminuria
$30-150 \mathrm{mg}$
2. Mild
3. Moderate
$150-500 \mathrm{mg}$
4. Heavy
5. Nephrotic range
$500-1000 \mathrm{mg}$
$1000-3000 \mathrm{mg}$
more than $3500 \mathrm{mg}$

As kidney disease progresses, more protein enters the urine. People with Nephrotic-range Proteinuria typically have extensive glomerular damage and usually develop Nephrotic syndrome.

Causes and risk factors: hypertension and diabetes are the two biggest risk factors for Proteinuria where formerly they had been excluded. In the U.S., diabetic nephropathy accounts for about $40 \%$ of new cases of ESRD, and in 1997, the cost for treatment of diabetic patients with ESRD was in excess of $\$ 15.6$ billion. About $20-30 \%$ of patients with type 1 or type 2 diabetes develop evidence of nephropathy, but in type 2 diabetes, a considerably smaller fraction of this progress to ESRD.

However, because of the much greater prevalence of type 2 diabetes, such patients constitute over half of those diabetic patients currently starting on dialysis. There is considerable racial/ethnic variability in this regard, with Native Americans, Hispanics (especially Mexican-Americans), and African-Americans having much higher risks of developing ESRD than non-Hispanic whites with type 2 diabetes.

\section{Etiology and prognostic significance of albuminuria in diabetes $^{6}$}

Persistent clinical proteinuria (i.e., urinary protein excretion greater than $0.5 \mathrm{~g} / 24 \mathrm{~h}$ ) is an ominous development in a person with diabetes. It eventually leads 
to a decline in the glomerular filtration rate and ultimately to end-stage renal failure or premature cardiovascular mortality. Progression of renal disease appears to be related to arterial blood pressure and protein intake and is primarily independent of the metabolic state.

More sensitive immunoassays for detecting low concentrations of albumin in urine have led to recognition of subclinical increases in albumin excretion rates in nonclinical proteinuria diabetic patients, a phenomenon named microalbuminuria. Studies have shown that patients with microalbuminuria have a significantly increased risk for clinical proteinuria and cardiovascular mortality. Microalbuminuria is rarely found during the first $5 \mathrm{yr}$ of a patient's diabetes, suggesting that it is a sign of early glomerular damage rather than a marker for susceptibility to it. In patients with non-insulin-dependent diabetes mellitus (NIDDM), an association has been found between microalbuminuria and coronary heart disease, but this relationship needs further investigation.

In patients with insulin-dependent diabetes mellitus (IDDM), this subclinical form of proteinuria is associated with poor metabolic control and, more important, with marginal elevation of blood pressure. Correction of hyperglycemia by intensified insulin treatment might arrest progression to persistent clinical proteinuria; moreover, restricted protein intake and lowering of blood pressure have been shown to reduce the albumin excretion rate.

Microalbuminuria in Diabetes Mellitus in University Hospital of West Indies ${ }^{7}$

Abstract: Micro albumin was determined on random urine specimens by an immunoassay method on 258 patients attending the Diabetic outpatients clinic at the University Hospital of the West Indies. Overall, there were $173(67.05 \%)$ patients with nephropathy. Of these, 150 $(58.14 \%)$ had microalbuminuria and $23(8.91 \%)$ had Microalbuminuria.

In the 68 patients with Type 1 diabetes 52(76.47\%) had nephropathy - 41(60.29\%) with microalbuminuria and $11(16.18 \%)$ with macroalbuminuria. In the 190 patients with Type 2 diabetes $121(63.68 \%)$ had nephropathy $109(57.37 \%)$ with microalbuminuria and $12(6.32 \%)$ with macroalbuminuria. In all groups over $70 \%$ had elevated Glycosylated Haemoglobin(HbA1c). This emphasizes the need for proper control of the diabetes.

\section{Rapid micoralbuminuria screening in type 2 diabetes mellitus: simplified approach with Micral test strips and specific gravity ${ }^{8}$}

Abstract: In this prospective study, a total of 444 urine samples of type 2 diabetic patients were obtained from the ABCD study cohort for analysis. Urinary albumin concentrations were determined using Micral test strips and compared to results measuring albumin by the immunoturbidimetry method of timed collections. Urine specific gravity was measured by a standard urine dipstick.
Results: The performance characteristics of the Micral test strips for detecting microalbuminuria $(30-300 \mathrm{mg}$ albumin/24 h) were adequate but not optimal: sensitivity $88 \%$, specificity $80 \%$, positive predictive value $69 \%$, negative predictive value $92 \%$. A concomitant specific gravity determination was useful in indexing the magnitude of false negative and false positive readings by the Micral test strips.

Conclusions: While the use of Micral test strips provides a rapid approach to detecting microalbuminuria in type 2 diabetic patients.

\section{The screening for diabetic nephropathy in diabetic clinic in Khartoum - Sudan'}

\section{Sid Ahmed Elhidir Ahmed and Mohammed Osman} Elhassan Gadour

Abstract: Ninety eight adult types 1 and 2 diabetic were studied using simple, direct, standardized questionnaire, previous records were seen and a urine sample for each patient was examined for proteinuria.

Results: $6.1 \%$ of the total number had urine examination on regular bases, $75.5 \%$ rarely had examination, while $18.4 \%$ had urine never been examined before in the diabetes clinics .None of patients was diagnosed as having diabetic nephropathy or seen by a nephrologist

Only $7.1 \%$ of the patients were using ACE inhibitors or $\mathrm{ARB}$ agents and these were prescribed for indications other than diabetic nephropathy. Testing urine of our patients we found that $18.4 \%$ had macroalbuminuria, $40.8 \%$ micralbuminuria, while $40.8 \%$ had negative results the majority of the patients with either type of albuminuria were in the age group 51-65 year and most of them had type 2 diabetes.

Conclusion: A large number of our patients had evidence of diabetic nephropathy. However none of them had been screened before for that. Despite the small number of patients, this study raises a serious alarm regarding the clinical practice in our diabetic clinics in Khartoum- Sudan and strongly recommends urgent intervention by the authorities to implement the international guidelines of screening and management of these patients.

\section{Diabetes mellitus associated with end stage renal disease in Sudanese patients ${ }^{10}$}

Done by Dr/Nazar Mohamed Abdel Rhman M. B. B. S Juba University

Abstract: A total of 856 patients diagnosed with ESRD on haemodialysis were retrospectively studied $68.2 \%$ of them are males $31.2 \%$ are females the most affected age group range between the $46-55$ years ,most of the study group of low education. The study found that the prevalence of DM among a group of ESRD $14.3 \%$.The mean age of them was $59.3 \%$ year the elderly are more 
affected ,male represent $76.2 \%$ and female $28.8 \%$ type 2 diabetes mellitus account $84 \%$ and type 1 diabetes account about $16 \%$.The mean duration between diagnosis of diabetes mellitus and the diagnosis of ESRD is 14.6+8.4 years . Hypertension is found as co factors in $56.6 \%$.The prevalence of other complications of diabetes mellitus in association with ESRD is found to be as $32 \%$ retinopathy and neuropathy $42 \%$.

\section{Material and Method}

\subsection{Research Design}

\section{Study Design}

This is a prospective cross - sectional study.

\section{Study Setting}

The Diabetic center in Omdurman Teaching Hospital and Altyser Health Center.

\section{Patients Definition}

Diabetic patients type I \& II with in age group (9-90) year old.

\section{Sample Size}

100 diabetic patients referred to diabetic center in Omdurman Hospital and Altyser Health Center

\section{Data Collection}

Data was collected by closed questionnaire after taking consent from the patients.

\section{Data Analysis}

Data was analyzed by computer using S.P.S.S. software.

\section{Data Presentation}

Information obtained will be presented graphically through tabulation and figures

\section{Study Area:}

Diabetic center in Omdurman Teaching Hospital, and Altyser health center in Omdurman city in Khartoum state capital of Sudan, containing primary health care providing vaccination and antennal clinic, diabetic clinic, minor theatre\& laboratory.

\section{Study Population}

All diabetic patients referred to diabetic center in Omdurman Hospital and Altyser Health Center during 6 month period from January 2009 up to June 2009.

The patients were called for interview written consent was be obtained.

Diabetic patients with type I \& II were randomly selected from patients attending the out patients diabetic clinic in Omdurman Teaching Hospital. And Altyser Health Center .According to following criteria: All patients were diabetic type I \& II with the age group (9-90) year old.

\section{Exclusion Criteria}

Patients with renal impairment

Pregnancy.

End stage renal damage.

Urinary tract infection.

Hypertension.

\section{Ethical Consideration}

Ethical clearance was obtained from the Sudanese Ministry of Health. The objectives of the study were explained to all individuals participating in the study, and all participants gave informed consent, the children taken the consent from their parents.

\section{Method of testing Microalbuminuria}

Chemstrip Micral $^{4}$ Test principle is an optically read immunoassay to detect albumin in urine samples. When the test strip is dipped into a urine sample, urine passes via a wick fleece into the conjugate fleece. Any albumin present in the sample binds itself specifically to the gold-labeled antibodies.

Excess antibody-gold conjugate is bound by immobilized albumin in a capture zone, so that the detection field is reached only by conjugate molecules charged with the urinary albumin. Depending on the albumin concentration, the detection field assumes a color ranging from white to red. The reaction time is one minute. The color is visually compared to the color blocks on a chart attached to the vial.

First the urine was examined for macroalbuminuria by using dipstick method. Patients with positive results were examined for other causes like urinary tract infection, hypertension, end stage renal disease ESRD,

Patients with negative results were further screened for microalbuminuria by using especial test for microalbuminuria dipstick method.

\section{The Results}

100 diabetic patients type I \& II were studied for microalbuminuria using dipstick those with more than $100 \mathrm{mg}$ were considered being positive for microalbuminuria.

\section{Gender Distribution}

Out of 100 diabetic patients $66 \%$ were females and $34 \%$ were males (Table 1, Figure 1) 
Distribution of patients according to residence

Table 1. Residence State in Sudan

\begin{tabular}{|c|c|c|}
\hline & Freq. & $\%$ \\
\hline Khartoum State & 92 & 92.0 \\
\hline Sinner State & 1 & 1.0 \\
\hline Blue Nile State & 1 & 1.0 \\
\hline Gezira State & 2 & 2.0 \\
\hline South & 1 & 1.0 \\
\hline Northern State & 2 & 2.0 \\
\hline Nahr ELNeel State & 1 & 1.0 \\
\hline Total & 100 & 100.0 \\
\hline
\end{tabular}

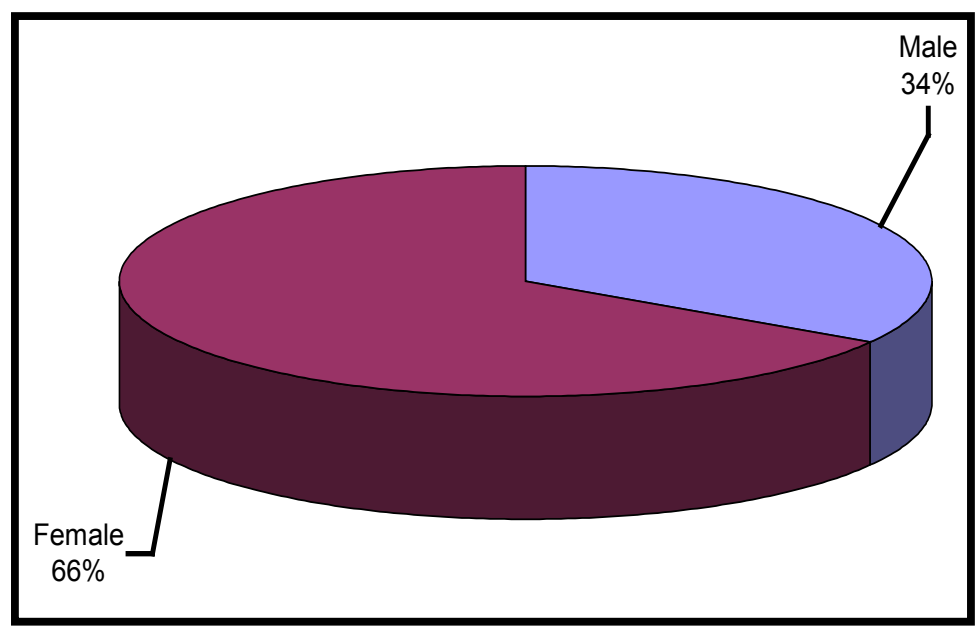

Figure 1. Show gender distribution

\section{Patients' distribution:}

Type I constituted 5\%, type II constituted 95\%, and the age range from $(9-90)$ year old (Figure 2)

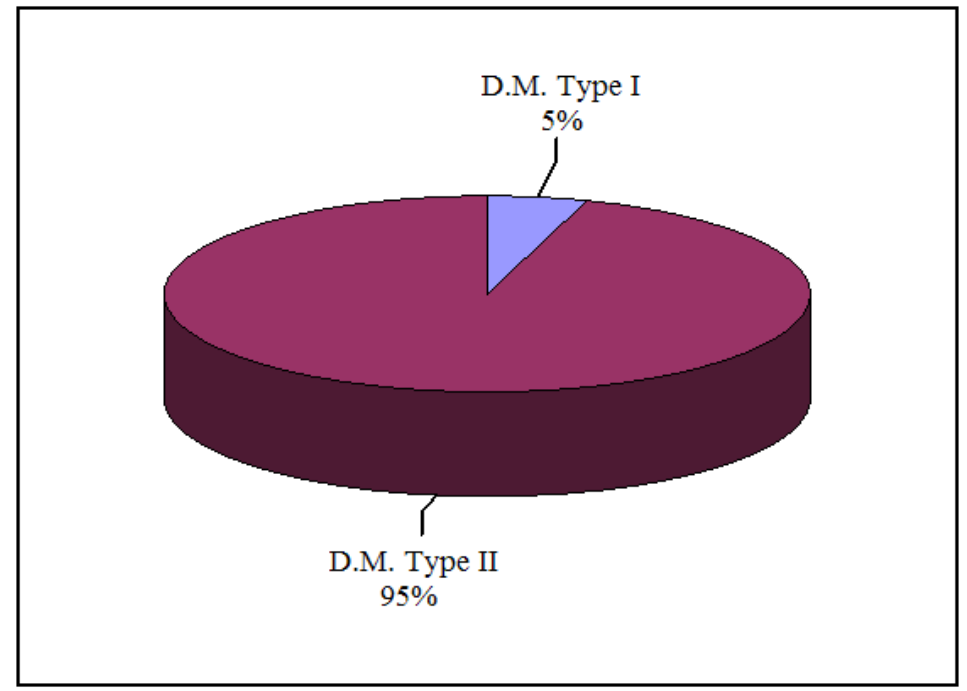

Figure 2. Show types of diabetes type I and type II 


\section{Age Distribution}

Most of the patients were more than 55 years of ages. The age of youngest patient was 9 years (Figure3).

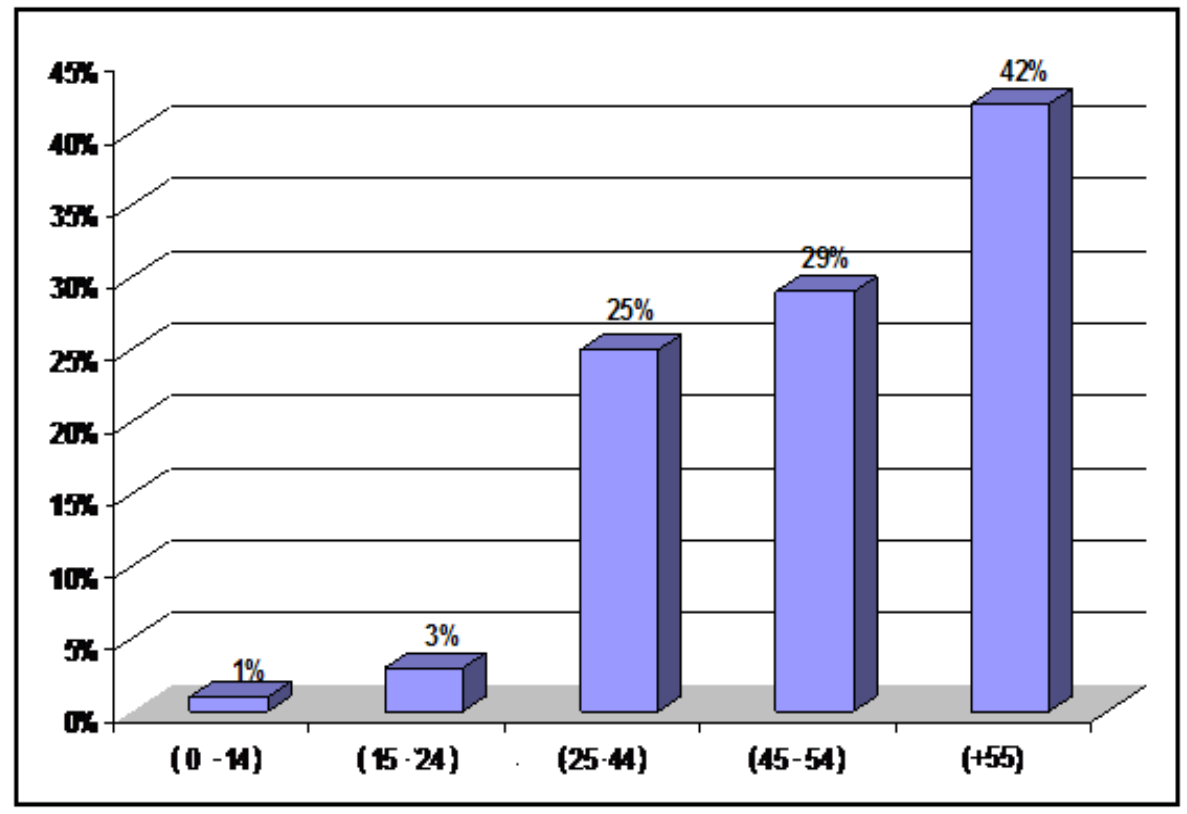

Figure 3. Show age group distribution

Results of the investigation of urine containing microalbuminuria:

Microalbuminuria was detected in $41 \%$ of the patient; most of the patients with microalbuminuria were in the age group (45-54). (Table2, Figure 4).

Distribution of patients according to the occupation most of them are house wife

\begin{tabular}{|c|c|c|}
\hline & Freq. & $\%$ \\
\hline House wife & 62 & 62.0 \\
\hline Worker & 15 & 15.0 \\
\hline Farmer & 1 & 1.0 \\
\hline Student & 3 & 3.0 \\
\hline Doctor & 1 & 1.0 \\
\hline Retired & 3 & 3.0 \\
\hline Clerk & 1 & 1.0 \\
\hline Engineer & 3 & 3.0 \\
\hline Driver & 2 & 2.0 \\
\hline Business & 7 & 7.0 \\
\hline Health visitor & 1 & 1.0 \\
\hline Midwife & 1 & 1.0 \\
\hline Not specified & 1 & 1.0 \\
\hline Total & 100 & 100.0 \\
\hline
\end{tabular}




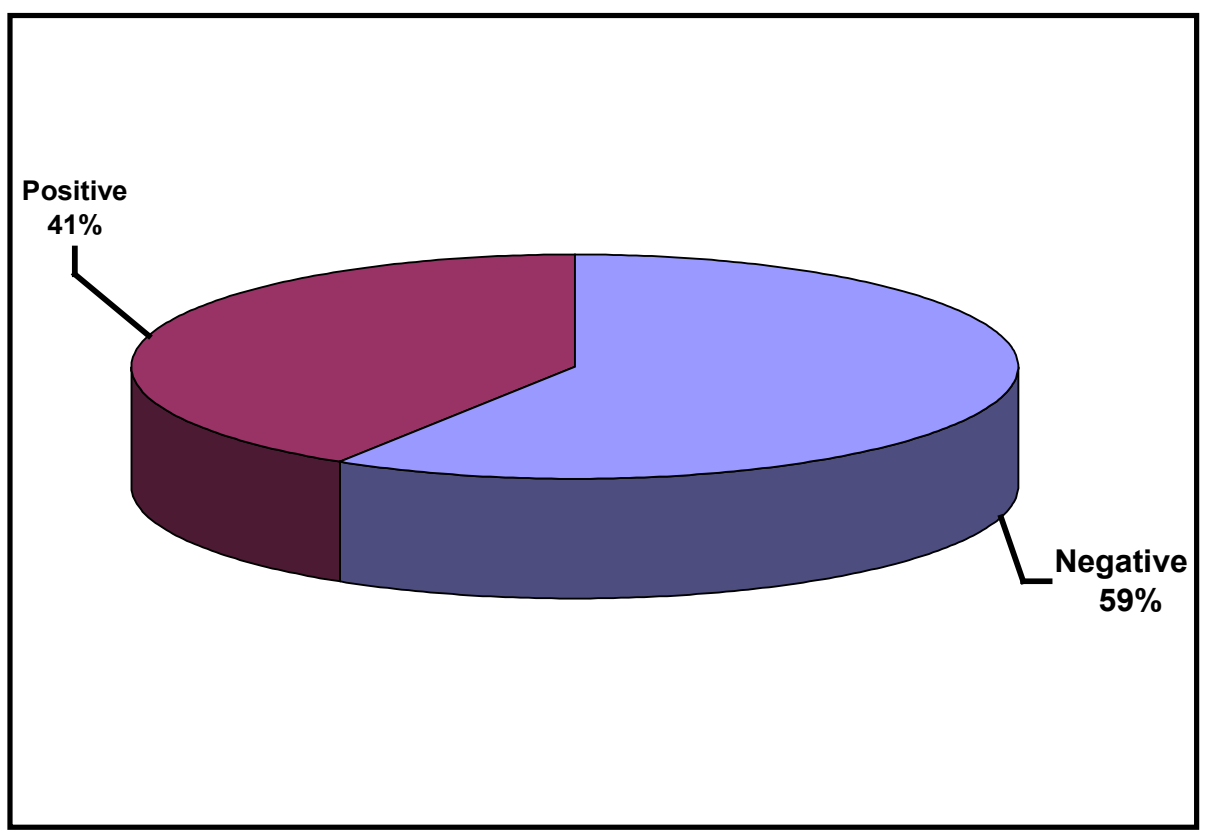

Figure 4. The result of the investigation of the urine containing microalbuminuria

Microalbuminuria in the females was $28 \%$, and the male's was $13 \%$

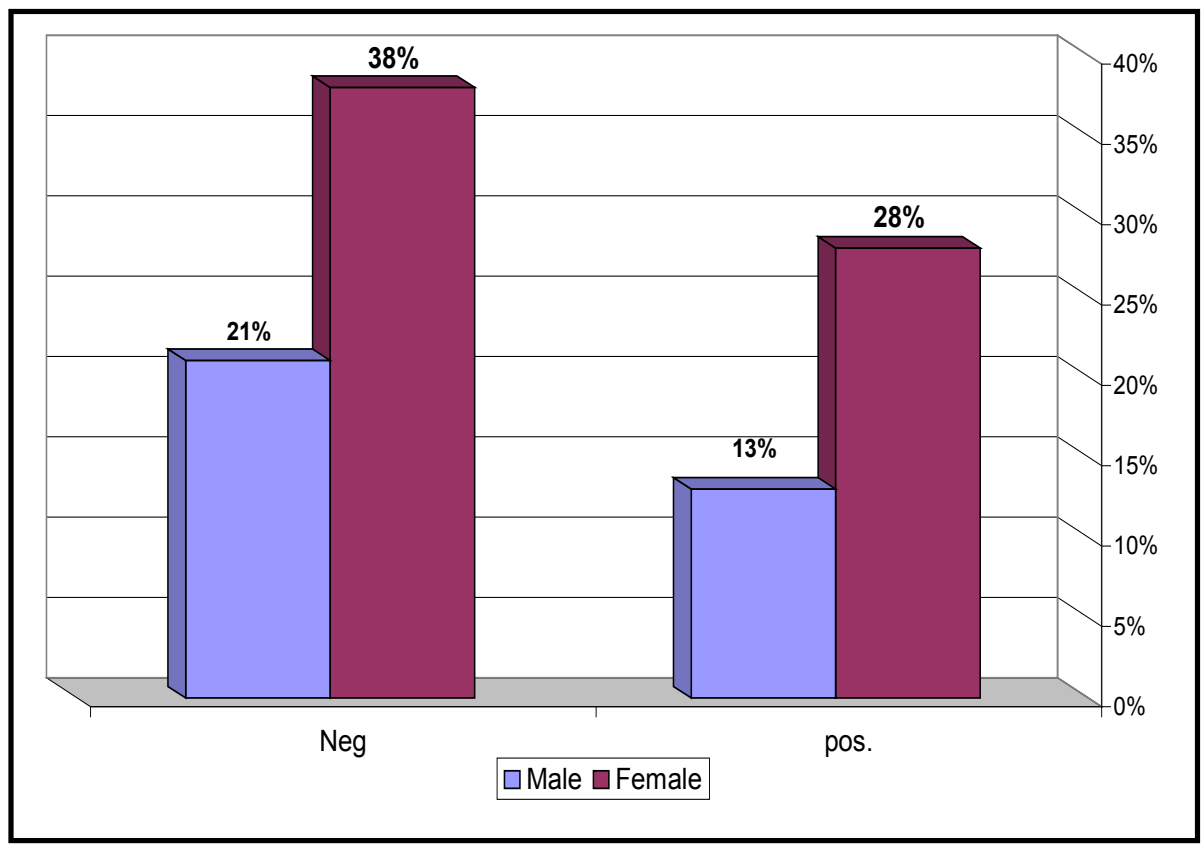

Figure 5. The result of the investigation of the urine containing micro albumin

Distribution of microalbuminuria in the urine according to age groups.

$\begin{array}{lll}- & (0-14) \text { age group } & 1 \% . \\ - & (15-24) / / & 2 \% . \\ - & (25-44) / / & 12 \% . \\ - & (45-54) \text { age group } & 9 \% . \\ -\quad(55+) / / & 17 \% .\end{array}$

- Most of the patients with positives microalbuminuria were the age group 55year and above

Distribution of microalbuminuria according to the duration of diabetes 


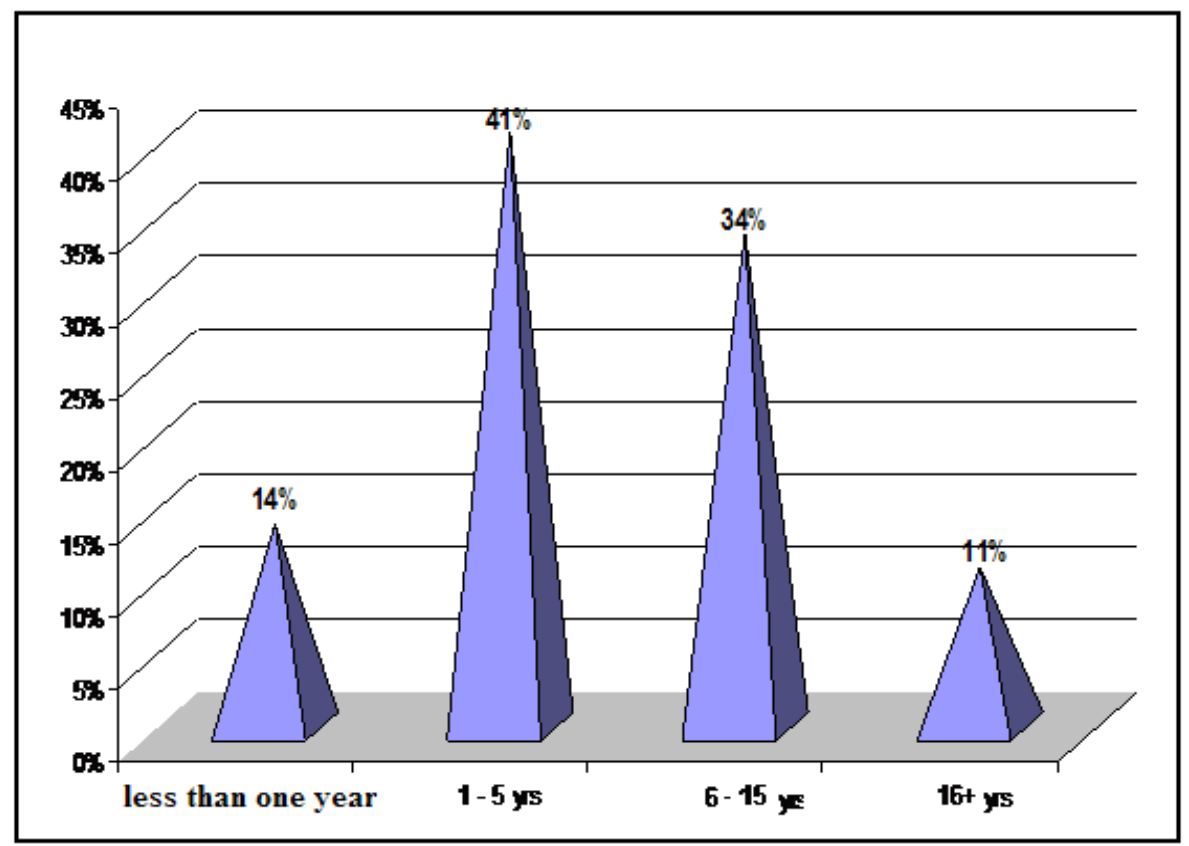

Figure 6. Duration of diabetes in relation to the presence of microalbuminuria

\section{Discussion}

In this study a total number of 100 diabetic patients were screened, for microalbuminuria using a special test .Out of 100 patients 74 seen in Omdurman Teaching Hospital and about 26 patients seen in Altyser Health center. In Altyser Health center the total number with positives microalbuminuria were 11 patients, 8 of them females and 3 males. Out of the 100 patients $66 \%$ were females and $34 \%$ were males. The ratio between them one third two third male to females.

Microalbuminuria was detected in $41 \%$ of the patients for the first time in, While $59 \%$ had negative results, $28 \%$ were females and $13 \%$ were male .Most of the patients with microalbuminuria were in group age (45 -54). In study done in Omdurman teaching hospital done by

Dr/ Sid Ahmed Elkhidir in Jan 2008 showed that out of 98 diabetic patients $40.8 \%$ with positive microalbuminuria8.

In this study we found that the majority of the patients with positive. Microalbuminuria were in the age group (4554) which slightly differs from study done by DR /Sid Ahemd Elkhidir .8

Another study done in the University of West indies Hospital showed that out of 258 patients $173(67.05 \%)$ patients had nephropathy and 150(58.14\%) had microalbuminuria and 23(8.91) had macroalbuminuria 7

The results of this study reflect the facts the diagnosis of diabetic nephropathy is missed due to the lack of proper screening.

No one of these patients with positive results specially in Omdurman Teaching Hospital received treatment ACEI. In study of Dr / Sid Ahemd, he found that only $5.6 \%$ of diabetic patients were using ACEI and these agents were prescribed for reasons other than diabetic nephropathy in all patients.

Large number of diabetic patients has abnormal protein excretion and need further tests to confirm the cause of the Proteinuria for proper management to prevent or slow their progression toward end stage renal failure. The clinical practice in Sudan is not following the international guidelines in screening diabetic nephropathy, while there is a simple method to discover the diabetic nephropathy by using dipstick strip method especially for microalbuminuria after excluding macroalbuminuria by simple way.

In Conclusion the clinical practice in diabetic clinics in Sudan is not following the international guidelines regarding the screening for diabetic nephropathy, so significant number of the diabetic patients with diabetic nephropathy are missed, The advantages of the test for microalbuminuria is simple, quick, cheap and reliable for detecting diabetic nephropathy. Proper management of microalbuminuria will prevent or slow the progression end stage renal failure. Using of urine strip for microalbuminuria will reduce the complications of diabetic nephropathy, should be used in all diabetic centers and hospitals in Sudan.

\section{REFERENCES}

[1] Davidson principle of medicine, 19 the Edition 2004, page 644.

[2] http://www.nephrologychannel.com/proteinuria/index.shtml Home » Proteinuria \& Microalbuminuria » Overview, Types, 
Causes and Risk Factors, Signs and Symptoms

[3] Original Date of publication 01 May 2001. Reviewed by .Stanley J. Swezevesk, 111, M. D

[4] Nephro 1 Dial Transplant (2004) 19: 1881-1885. Nephrol Dial Transplant Vol. 19 No. 7 (C) ERA-EDTA 2004; all rights reserved. http://www.Roche-Diagnostic, us/ products - service /Micralhtm

[5] American Diabetes Association .Standards of medicine care in diabetes- 2009, Diabetes care. January 2009; 32:S13-S61.

[6] Nephrology Dialysis Transplantation, March 1, 2005, 20:417-508. http:// care .diabetes journals. Org/content/11/10/840 abstract.

[7] American Association for clinical medicine. Clinical Chemistry, Vol 38 ,588- 891, Copyright 1992

[8] Nephrology Dialysis transplantation, Vol 112, Issue 178-80, copyright 1997 by oxford university press.

[9] Sid Ahmed Elhidir Ahmed and Mohmmed Osman Elhassan Gadour Sudan Medical specialization Boards council of internal medicine, research library.

[10] Nazar Mohamed Abdel Rahman, Sudan Journal of Medical Sciences vol. 3, No. 4, Dec 2008, page. 285-290. 